\title{
Psychotherapy Training for Psychiatric Trainees
}

\author{
Hugh КосH, Senior Clinical Psychologist, Whitchurch Hospital, Cardiff and \\ RuChard SCORER, Lecturer in Psychological Medicine, University Hospital of Wales, Cardiff
}

'Our first concern must be to improve the psychotherapeutic skills of psychiatrists in general, as a means of providing a better service to our patients.' Thus, Sydney Brandon concluded his personal view on psychotherapy in the Bulletin last year (July 1982, 6, 120). It was a similar concern that motivated us, two years ago, to set up a scheme which initially aimed to provide trainees in psychiatry with some form of regular teaching, experience and supervision in dynamic psychotherapy.

The stimulus to set up this scheme arose from at least four sources: (a) Requests came from trainees for 'teaching' in psychotherapy. At times these were vague and occasionally seemed to have more to do with the Membership examination and an awareness of a gap in knowledge and experience. (b) The College has recommended that clinical experience of psychotherapy be obtained prior to taking the MRCPsych examination, including 'continuity of experience with particular patients over at least two years' (Royal College of Psychiatrists, 1978). (c) There was an awareness of the needs of some patients where trainees may have embarked on 'psychotherapy' having had no teaching, under minimal supervision and with little idea of techniques or problems involved. (d) We were aware that the 'medical model' exerted a profound effect on many trainees' clinical practice; patients falling into this model would often be referred back to their GPs with little prospect of obtaining psychotherapeutic help.

\section{Aims}

Our broad aims have been to increase trainees' sensitivity to the interpersonal aspects of therapist/patient communication and their ability to facilitate change through the therapeutic relationship. By discussing a variety of therapeutic approaches, trainees have been invited to develop their own individual ways of working (Smail, 1978).

Our aims can be stated as follows:

(1) To provide trainees from their earliest placements with the opportunity to learn about, practise, and be supervised in individual psychotherapy. In using the term psychotherapy we refer specifically to dynamic psychotherapy, but include basic interviewing skills and concentrate on offering experience in goal-orientated, timelimited, individual therapy, lasting for up to six months.

(2) To provide similar experience for trainees in clinical psychology, and qualified nurses with an interest in psychotherapy, and others from related caring professions.

(3) Through a weekly supervision group, to give the trainees understanding of small-group dynamics.

(4) To encourage self-awareness among trainees, together with a recognition that their attitudes to, and relationships with patients, may reflect attitudes to, and relationships with colleagues, friends and family.

(5) To provide a regular forum for teaching and discussion of the theoretical foundations of psychotherapy, its historical development, its various goals, and research into its efficacy as a form of treatment.

It is not implied that trainees who have completed a sixmonth course have thereby become competent psychotherapists or gained comprehensive knowledge, but we hope that trainees will have learned how to construe a patient's problem broadly, to proceed sensitively in their contact with patients, and to recognize the value of psychotherapeutic principles in relationships with staff and patients, while at the same time having a realistic evaluation of their own personal therapeutic resources.

\section{Methods}

Trainees are expected to commit themselves to the training scheme for a period of six months. On the Cardiff Rotational Training Scheme this time will usually coincide with that of their placement on one particular unit. We have kept to a maximum of eight trainees with two supervisors (the authors).

The trainees in the scheme have usually been those at the beginning of their psychiatric training. It is all too common to find that trainces in their early months in psychiatry will be more familiar with first-rank symptoms of schizophrenia and dosages of anti-depressants than they will about interviewing techniques and the potential of their own personality in therapy.

As soon as possible after joining the scheme, each trainee is given a patient who has been assessed by one of us as being suitable for time-limited psychotherapy. Trainees will see their patients for a weekly 50-minute session (i.e. a total of approximately 20 sessions). They are asked to keep a case file with brief notes on each session.

A weekly group of $1 \frac{1}{2}$ hours duration provides the regular meeting point for supervisors and trainees. Two trainees are specifically given time each week to report on a recent session, and to provide material for discussion. (Others have the opportunity to raise critical issues from their therapy case to be dealt with more briefly.) Our expectations of the trainees are specified for them in writing and the following are required: (a) A written summary presenting problems and relevant developmental history should be provided for each group member. (b) A formulation should be made which should include some tentative views about the nature and development of the problems, type and course of 
therapy, and include some criteria for change which might be assessed during, and at the end of therapy. This may be made with the help of the group as a whole, or one of the supervisors. (c) Videotape and/or audiotape recordings will be used to present material from a recent therapeutic session, as well as verbatim accounts. (d) Supervision includes clarification of the trainees' understanding of what the patient is communicating to them, the trainees therapeutic plan, his interventions, and the results of his interventions. (e) The trainee is required to provide feedback at the end of sessions as to the usefulness of supervision given. (f) A trainee should have some familiarity with the patients being seen by other trainees, and to this end it is encouraged that notes are made during the supervision group.

With up to eight trainees it is clear that supervision within the group is only possible every three or four weeks. Individual supervision is available on a regular basis with one of the two supervisors. A monthly afternoon workshop is held for more formal teaching on such topics as interview skills, defence mechanisms, transference and countertransference and research in psychotherapy.

\section{Problems in running the scheme}

There are limitations as to what can be achieved in a period of six months. Not being full-time psychotherapists we have other commitments, but have felt that the course might be more satisfactory were it possible to offer greater supervision time, and more in the way of theoretical foundations via seminars. Trainees have varied attitudes to the course, but in two and a half years only one has dropped out altogether, and he was a GP trainee who felt that the course was not relevant for him. They have developed an increasing enthusiasm and many have asked for continued involvement after the six-month period.

Personal problems: Personal problems have arisen because of trainees' attitudes (often defensive in nature) which have impaired their interest in therapy and their motivation for supervision, with inevitable effects on the patient. These have included a rigid adherence to a medical model where all problems are viewed in terms of illness.

Many have found it difficult to get away from the concept of an active therapist 'doing things to' the passive, grateful patient. There have been suspicions about the nature of psychotherapy with its inherent uncertainties, and this has led some trainees to focus discussion around demands for proof that psychotherapy is effective, before being prepared to take on patients. There has been a reluctance among trainees to explore and acknowledge their own feelings, both positive and negative. An unwillingness to see themselves as the major component in therapy has been combined with a reluctance to take credit for positive aspects in therapy. A recurring assumption has been that there was a 'right thing' and a 'wrong thing' to say in response to a particular comment by a patient. This has clearly inhibited spontaneity and genuineness in therapy and inevitably trainees may have felt that they should model themselves only on their supervisors, and not accept responsibility for their own statements, their own behaviour, and their responses in therapy.

Practical problems: All the trainees involved have been working on the Rotational Training Scheme on an acute admission ward where service commitment has been unpredictable and at times demanding. For valid reasons they may have found it impossible to attend the supervision group as regularly as was desirable. Trainees in psychotherapy need space and time, and these are not readily available for trainee psychiatrists (Burrell, 1982).

\section{Planned developments}

As with most training schemes like this, after each sixmonthly period of supervision has been completed, new ideas are implemented to improve the efficiency of the learning process. The following innovations are planned in the areas of initial assessment, evaluation of supervision and increasing clarification of interpretative skills.

In order that trainees may immediately be encouraged to examine the many important categories of general psychological, and then specific psychodynamic assessment, it is our intention to provide trainees with an interview booklet outlining categories of importance to guide the initial interviews. These may help purely to direct the interviewer's attention, or they may serve to indicate new areas for discussion or clarification.

Evaluation of supervision has been discussed by many authors (e.g. Hess, 1980). However, criteria for evaluation differ, depending on the trainee's level of psychological mindedness and the client group. We are trying to clarify suitable criteria for evaluation by firstly asking the trainees themselves to feed back suggestions or interpretations made in supervision which they found useful. The trainers also keep a record of comments thought to be of value. A method of focusing on relevant areas of supervision will then be devised to carry out a more systematic evaluation.

Micro-skills training of psychotherapeutic skills has been discussed by Ivey (1971). A workshop for training in basic interviewing skills is already part of the programme. The parts of this workshop relating to 'Reflection of Feeling' and 'Summarization and Paraphasing' are being elaborated to include 'Clarification and Interpretation' skills.

REFERENCES

BURRELL, M. (1982) Training needs in psychotherapy. Bulletin of the Royal College of Psychiatrists, 6, 9.

Hess, A. K. (1980) Psychotherapy Supervision: Theory, Research and Practice. New York: Wiley.

IVEY, A. (1971) Micro-counselling: Innovation in Interviewing and Training. Springfield, Illinois: Thomas.

Royal College of Psychiatrists (1978) Educational Programmes for Trainees in Psychiatry. (Leaflet circulated from the College's Examinations Office.)

Small, D. J. (1978) Psychotherapy: A Personal Approach. London: Dent. 